Reprod. Nutr. Dévelop., 1988, 28 Suppl. $\mathrm{n}^{\circ} 1,99-100$

\title{
Influence de l'actaplanine sur la digestion d'un ensilage de maïs, de ray-grass vert et de tourteau de soja, mesurée en Rusitec
}

\author{
Christine GUY $\left({ }^{*}\right)$, J. P. JOUANY
}

Station de Recherches sur la Nutrition des Herbivores,

Unité de la Digestion Microbienne, I.N.R.A.,

Theix, 63122 Ceyrat, France.

$\left(^{*}\right)$ Université Blaise Pascal, Laboratoire de Génie Chimique Biologique, 63170 Aubière.

Summary. Actaplanin increased propionate percentage at the expense of acetate and butyrate, and lowered the amount of methane in gases produced in a Rusitec fermenter. It had no major effect on the other end products nor on the digestion of rye grass, soybean meal or maize silage.

L'actaplanine (produit par la Sté Eli Lilly) est un antibiotique ayant des propriétés proches de la monensine. Son emploi en tant qu'additif alimentaire n'a fait l'objet que de deux courtes communications. La première (Chase et al., 1984) a mis en évidence un effet positif de cette molécule sur la croissance des génisses $(+80 \%)$ à la dose de $900 \mathrm{mg} / \mathrm{j}$. La deuxième (Clapperton et al., 1986) a montré que l'addition de $1 \mathrm{~g}$ d'actaplanine par jour diminuait à la fois les teneurs du lait en matières grasses $(-21,5 \%)$ et en protéines $(-6 \%)$, la production laitière des vaches n'étant pas modifiée. Dans les deux cas, la proportion d'acétate et de butyrate dans le mélange d'acides gras volatils a été réduite.

Nous présentons ici une étude concernant l'effet de l'actaplanine sur la dégradation de 3 aliments mesurée en fermenteurs Rusitec : ray-grass vert (RG), ensilage de maïs (EM), tourteau de soja (TS).

Matériel et méthodes. Six fermenteurs semi-continus de type Rusitec (volume de 1 litre), ensemencés avec un inoculum homogène provenant de 6 moutons adultes nourris avec une ration mixte, ont été utilisés. Ils recevaient chaque jour $17 \mathrm{~g}$ de matière sèche $(\mathrm{MS})$ de l'un des aliments placé sous forme broyée (grille de $4 \mathrm{~mm}$ ) dans des sachets de $18 \times 8 \mathrm{~cm}$ ayant des mailles de $50 \mu \mathrm{m}$. Deux fermenteurs étaient utilisés pour chaque aliment: un fermenteur témoin (FT) et un autre dans lequel 88 ppm d'actaplanine étaient incorporés dans l'aliment ( $\mathrm{FAC}$ ). Une solution tampon additionnée d'oligo-éléments et de sulfate de $\mathrm{Na}$, et contenant de l'urée $(1,2 \mathrm{~g} / \mathrm{j}$ pour $\mathrm{EM}$ et $0,6 \mathrm{~g} / \mathrm{j}$ pour $\mathrm{RG}$ ) ou du glucose $(1,5 \mathrm{~g} / \mathrm{j}$ pour TS) a été infusée en continu avec un taux de dilution journalier de 0,7 . Chaque période de mesure a duré 3 semaines : une semaine d'adaptation et 2 autres de mesures quotidiennes. Nous avons déterminé les principaux paramètres fermentaires de 1,5 à 2 heures après l'alimentation des fermenteurs et avons mesuré la part d'azote bactérien libre en dosant l'azote sur le culot de bactéries libres isolé par centrifugation fractionnée (Jouany et Thivend, 1972).

La quantité de matière sèche apparemment disparue (MSAD) et le taux de disparition des parois cellulaires (NDF) et de la lignocellulose (ADF) ont été 
calculés par pesée des sachets avant et après un séjour de $48 \mathrm{~h}$ dans les fermenteurs et analyse du contenu des sachets. La quantité de matière organique fermentée (MOF) a été calculée à partir de la production d'acides gras volatils (AGV).

Résultats et discussion (tabl. 1). La présence d'actaplanine n'a pas modifié les quantités de matière sèche apparemment disparue et de matière organique fermentée. Elle n'a pas non plus influencé la dégradation des parois cellulaires et de la lignocellulose, ni la production totale d'AGV ou la valeur du $\mathrm{pH}$. En revanche, l'actaplanine a eu un effet significatif sur la composition du mélange d'AGV pour les 3 substrats étudiés. La proportion de propionate a augmenté de $15 \%$ avec le tourteau de soja et de $75 \%$ avec l'ensilage de maïs, aux dépens de l'acétate et du butyrate. L'actaplanine n'a pas eu d'effet sur la production totale de gaz, mais elle a provoqué une diminution significative de la méthanogenèse qui est conforme à la modification de la composition du mélange des AGV.

La quantité d'azote bactérien présente dans la phase liquide du Rusitec n'a pas été influencée par l'actaplanine. Compte tenu de l'absence d'effet de cette molécule sur la dégradation de la matière organique des aliments, il est probable que la biomasse microbienne adhérente n'a pas non plus été modifiée. En valeur absolue, la mesure de l'azote bactérien associé à la phase liquide ne correspond pas à la protéosynthèse microbienne totale, toutefois sa variation permet d'estimer l'effet de l'actaplanine sur ce paramètre.

TABL. 1. - Paramètres digestifs dans les fermenteurs témoins (F.T.) et les fermenteurs recevant l'actaplanine (F. Ac).

\begin{tabular}{|c|c|c|c|c|c|c|}
\hline \multirow{2}{*}{ Paramètres } & \multicolumn{2}{|c|}{$\begin{array}{l}\text { Ensilage de } \\
\text { maïs }\end{array}$} & \multicolumn{2}{|c|}{$\begin{array}{l}\text { Ray-grass } \\
\text { vert }\end{array}$} & \multicolumn{2}{|c|}{$\begin{array}{l}\text { Tourteau de } \\
\text { soja }\end{array}$} \\
\hline & F.T. & F. Ac & F.T. & F. Ac & F.T. & F. Ac \\
\hline $\operatorname{MSAD}(\mathrm{g} / \mathrm{j})$ & 9,83 & 9,94 & 13,0 & 13,0 & 11,0 & 11,5 \\
\hline MOF fermentée $(\mathrm{g} / \mathrm{j})$ & 8,32 & 7,72 & 5,9 & 6,1 & 7,2 & 8,4 \\
\hline Dégradation NDF (\%) & 23,6 & 26,3 & 59,9 & 57,3 & - & - \\
\hline Dégradation ADF (\%) & 23,3 & 25,0 & 58,3 & 55,2 & - & - \\
\hline $\mathrm{pH}$ & 6,7 & 6,6 & 6,9 & 6,9 & 6,9 & 6,9 \\
\hline $\operatorname{AGV}(\mathrm{mM} / \mathrm{j})$ & 61,1 & 62,1 & 50,3 & 52,5 & 62,6 & 75,6 \\
\hline $\mathrm{C}_{2} / \mathrm{C}_{3}$ & 4,5 & $2,1^{* *}$ & 2,4 & $1,9^{* *}$ & 3,2 & $2,6^{*}$ \\
\hline Gaz (I/j) & 2,6 & 2,5 & 2,1 & 2,2 & 2,3 & 2,9 \\
\hline $\mathrm{CO}_{2} / \mathrm{CH}_{4}$ & 5,0 & $7.8^{*}$ & 5,5 & 5,7 & 1,8 & $3,0^{*}$ \\
\hline $\mathrm{N}-\mathrm{NH}_{3}(\mathrm{mg} / \mathrm{j})$ & 102,5 & 73,0 & 273,9 & 251,2 & 889,2 & 872,4 \\
\hline $\begin{array}{l}\text { Azote bactérien de la phase liquide } \\
(\mathrm{mg}-\mathrm{Nb} / \mathrm{j})\end{array}$ & 62 & 59 & 87 & 91 & 79 & 96 \\
\hline $\begin{array}{l}\text { Efficacité de la synthèse bactérienne } \\
(\mathrm{gNb} / \mathrm{kg} \mathrm{MOF})\end{array}$ & 7,4 & 7,6 & 14,7 & 14,9 & 11,0 & 11,4 \\
\hline
\end{tabular}

Les différences sont significatives au seuil $P<0,05^{*}$ ou $0,01^{*}$ *

Remerciements. - Ce travail a été réalisé grâce à l'aide financière de la Société Lilly France, 203, Bureaux de la Colline, Saint-Cloud (France).

Chase C. C., Randel R. D., Carroll L. H., 1984. J. anim. Sci., 59, 33A.

Clapperton J. L., Steele W., Banks W., 1986. Proc. Nutr. Soc., 46, 43A.

Jouany J. P., Thivend P., 1972. Ann. Biol. anim. Bioch. Biophys., 12, 673-677. 